\title{
In-Host Adaptation of Salmonella enterica Serotype Dublin during Prosthetic Hip Joint Infection
}

\author{
Faten El Sayed, Guillaume Sapriel, Nizar Fawal, \\ Aurelia Gruber, Thomas Bauer, Beate Heym, \\ Caroline Dupont, Henri-Jean Garchon, \\ Jean-Louis Gaillard, Martin Rottman, \\ Simon Le Hello
}

Genome degradation has been central to the adaptation of Salmonella enterica serotypes to their hosts throughout evolution. We witnessed the patho-adaptation of a strain of Salmonella Dublin (a cattle-adapted serotype) to a human host during the course of a recurrent prosthetic hip joint infection evolving over several years.

\begin{abstract}
Calmonella enterica serotype Dublin is a host-adapted bacterium with cattle as a predominant reservoir and is responsible for invasive, potentially life-threatening infections in humans $(1,2)$. In France, the epidemiology of Salmonella Dublin infections among humans corroborates surveillance data from the United States (1). Salmonella Dublin causes substantially more bloodstream infections and hospitalizations than other Salmonella serotypes. It is also much more likely to be isolated in metastatic foci of infection secondary to bacteremia. The prevalence of prosthetic joints in Salmonella Dublin patients is significantly greater than for other Salmonella cases (Table).

Host adaptation is central to pathogen evolution and is associated with gene acquisition, genome degradation (gene inactivation or deletion), or both. Genome degradation has played a major role in the adaptation of $S$. enterica serotypes Typhi and Paratyphi A to the human host throughout evolution (3). Degradation also has been recently reported in a severely immunocompromised patient in whom recurrent systemic Salmonella Enteritidis infections developed over 15 years (4). We report the phenotypic and genomic changes
\end{abstract}

Author affiliations: University of Versailles Saint-Quentin, Montigny-le-Bretonneux, France (F. El Sayed, G. Sapriel, B. Heym, H.-J. Garchon, J.-L. Gaillard); Hôpital Ambroise Paré, Boulogne-Billancourt, France (F. El Sayed, A. Gruber, T. Bauer,

B. Heym, C. Dupont, J.-L. Gaillard, M. Rottman); Atelier de Bioinformatique, Paris, France (G. Sapriel); Institut Pasteur, Paris (N. Fawal, S. Le Hello); Hôpital Raymond Poincaré,

Garches, France (M. Rottman)

DOI: https://doi.org/10.3201/eid2412.180214 undergone by Salmonella Dublin throughout a recurrent prosthetic hip infection in an immunocompetent patient.

\section{The Study}

In May 2011, a 74-year-old woman with bilateral hip prostheses (implanted in 1998 [right] and 2001 [left]) was admitted to intensive care for sepsis and left hip pain (online Technical Appendix Figure, https://wwwnc.cdc.gov/EID/ article/24/12/18-0214-Techapp1.pdf). Blood cultures and a joint aspiration yielded pure cultures of Salmonella spp. She underwent debridement and implant retention surgery, followed by a 6 -week intravenous course of cefotaxime and ciprofloxacin. Three years later, she sought care at the emergency department with acute-onset fever and prosthesis joint infection of the right hip and underwent right hip debridement and implant retention surgery. Blood cultures, joint aspirates, and all interoperative periprosthetic tissue samples yielded Salmonella spp. The patient received 2 weeks of intravenous amoxicillin and oral ofloxacin, was discharged, and received oral antimicrobial drugs for 10 more weeks. Six weeks after surgery, the surgical wound was healed, and the patient walked normally. One year later (2015), her primary care physician referred her to the hospital because of night fevers without local signs or implant dysfunction. Radioleucoscintigraphy showed right hip inflammation. Bilateral hip biopsies were performed, and the right hip biopsy sample tested positive for Salmonella spp. A right hip 1-stage exchange procedure was performed. All intraoperative periprosthetic tissue samples yielded Salmonella spp. A 6-week course of intravenous therapy with ceftriaxone and ciprofloxacin was administered. One year later (2016), the patient appeared to be free from infection and walked normally.

We characterized isolates by matrix-assisted laser desorption/ionization time-of-flight mass spectrometry (Bruker Daltonik GmbH, Bremen, Germany) and serotyping (French National Reference Center for Salmonella). We assessed carbohydrate metabolic activity using the API50CH system (bioMérieux, Marcy-l'Etoile, France) and biofilm formation using crystal violet (5). Antimicrobial susceptibility testing was performed using 2015 EUCAST (European Committee on Antimicrobial Susceptibility Testing) guidelines (http:// www.eucast.org/clinical_breakpoints/). We conducted high-throughput whole-genome sequencing using the Illumina NextSeq 500 system (Illumina, San Diego, CA, USA). 
Table. Demographic characteristics of patients and sources for Salmonella enterica serotype Dublin and other Salmonella isolates, France, 2010-2016

\begin{tabular}{|c|c|c|c|}
\hline Patient characteristic & Salmonella Dublin, no (\%) & Other Salmonella, no. (\%) & $p$ value ${ }^{*}$ \\
\hline All cases, $\mathrm{N}=63,264$ & 642 & 62,622 & \\
\hline \multicolumn{4}{|l|}{ Sext } \\
\hline $\mathrm{F}$ & 266 & 30,762 & $<0.01$ \\
\hline M & 363 & 30,401 & \\
\hline \multicolumn{4}{|l|}{ Age group, y } \\
\hline$<1$ & $4(0.6)$ & $3,767(6.0)$ & $<0.01$ \\
\hline $1-5$ & $33(5.1)$ & $15,302(24.4)$ & $<0.01$ \\
\hline $6-14$ & $19(3.0)$ & $8,636(13.8)^{\prime}$ & $<0.01$ \\
\hline $15-64$ & 199 (31.0) & $23,653(37.8)$ & NS \\
\hline$\geq 65$ & $375(58.4)$ & $9,801(15.7)$ & $<0.01$ \\
\hline Ūnknown & $12(1.9)$ & $1463(2.3)$ & NS \\
\hline \multicolumn{4}{|l|}{ Type of human sample§ু } \\
\hline Feces & $218(34.0)$ & $55,824(89.1)$ & $<0.01$ \\
\hline Blood & $264(41.1)$ & $3,268(5.2)$ & $<0.01$ \\
\hline Urine & $72(11.2)$ & $2,257(3.6)$ & $<0.01$ \\
\hline Articular & $20(3.1)$ & $30(0.05)$ & $<0.01$ \\
\hline Pus & $11(1.7)$ & $65(0.1)$ & $<0.01$ \\
\hline Bile & $1(0.2)$ & $32(<0.1)$ & NS \\
\hline Cerebrospinal fluid & 0 & $23(<0.1)$ & NS \\
\hline Other & $56(8.7)$ & $1123(1.8)$ & $<0.01$ \\
\hline Hospitalization & $368(57.3)$ & $20,556(32.8)$ & $<0.01$ \\
\hline \multicolumn{4}{|c|}{ 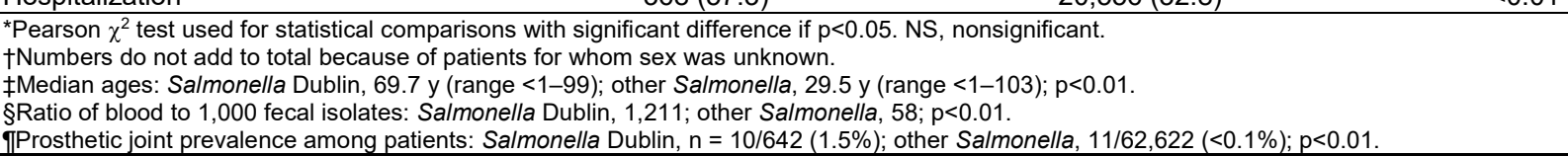 } \\
\hline
\end{tabular}

For each isolate, the paired-end reads were aligned against 2 Salmonella Dublin CT_020221853 and 3246 reference genomes to increase the single-nucleotide polymorphism (SNP) detection (GenBank accession nos. CP001144.1 and CM001151.1, respectively) (6) (online Technical Appendix). We annotated putative coding sequences using GeneMark (7) and performed ortholog identification, based on the prototypal human reference genome CT_020221853, using BLAST $+(8)$ with a $1 \times 10^{-10}$ E-value threshold and confirmed by a synteny conservation check. We first identified candidate pseudogenized sequences using a proteome/ proteome BLASTp approach (https://blast.ncbi.nlm.nih.gov/ Blast.cgi): protein sequences of strains Str.2011, Str2014, and Str 2015 with Query Coverage $<80 \%$ relative to the reference strain orthologs were selected as potentially pseudogenized sequences. We then analyzed nucleic acid level sequences corresponding to the previously selected candidates to confirm the change in coding sequences, and to precisely determine the genetic event kind of nucleic acid change involved (frame-shift mutation or indel).

We studied 3 patient strains: Str.2011 (May 2011, left hip intraoperative periprosthetic tissue [HIPT]), Str.2014 (April 2014, right HIPT), and Str.2015 (June 2015, right HIPT). Unlike Str.2011, Str.2014 and Str.2015 were nonmotile with no detectable $\mathrm{H}$ antigen and had lost the ability to use 9 of the 18 carbohydrates used by Str.2011, with slight profile variations (Figure). All strains could form biofilms and had identical wild-type antimicrobial drug susceptibility profiles and unchanged MICs for $\beta$-lactams and quinolones, without mutations in $\operatorname{gyr} A, \operatorname{gyr} B, \operatorname{par} C$, and parE genes. All 3 strains belonged to the sequence type 10 Salmonella Dublin population (http://mlst.warwick.ac.uk/mlst/dbs/Senterica). The mapping of Str.2011, Str.2014, and Str.2015 genomes against the Salmonella Dublin CT 020221853 and str.3246 reference genomes detected 451 and 268 SNPs, respectively, whereas the 3 patient strains differed by only 6 SNPs, strongly suggesting they derived from a single infecting strain. Four SNPs were nonsynonymous in coding genes; 2 SNPs were in an intergenic region (Figure).

Comparative proteome/proteome analysis detected putative pseudogenized sequences that were due to indels within 3 coding sequences in Str.2014 and Str.2015. These pseudogenes were involved in carbohydrate transport (ptsA) (14-bp deletion), lipopolysaccharide (LPS) biosynthesis (waaY) (16-bp insertion), and protein synthesis (tufB) (790-bp deletion, total gene deletion); we identified no pseudogene among the genes of the flagellar regulon. The pseudogene involved in carbohydrate transport, pts $A$, controls the import of carbohydrates, such as mannose, fructose, and N-acetyl-glucosamine (9), 3 carbohydrates used by Str.2011 but not Str.2014 or Str.2015. Mutation of the LPS biosynthesis gene waaY in Str.2014 and Str.2015 had apparently no effect on the expression of the Salmonella Dublin $\mathrm{O}$ antigen but might have affected flagellar assembly and function, as described for Salmonella Typhimurium and Escherichia coli $(10,11)$. The mutation of tufB in Str.2014 and Str.2015 leads to a one third decrease in the production of the translation elongation factor EF-Tu (the other two thirds is synthetized from tufA) (12). 
The patient in this study had no detectable gallbladder or intestinal carriage, which has been associated with recurrent Salmonella infections (13). She had no apparent immunodeficiency, a well-documented risk factor for invasive Salmonella Dublin infection. Salmonella Dublin most likely persisted as a biofilm at the surface of the implants, leading to recurrence of chronic disease despite prolonged antimicrobial therapy until the ablation of the infected material (14). The genomic changes we observed involved carbohydrate metabolism and LPS biosynthesis,
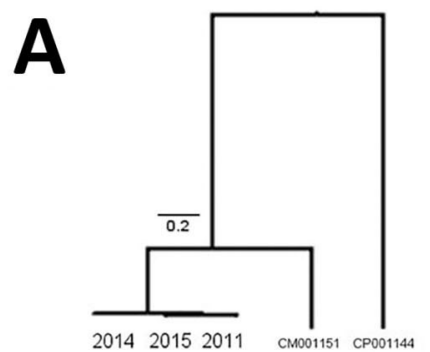

GCT GCT GAT D-mannonate oxidoreductase (SeD_A1830)

GAC GCG GAC Lytic enzyme (SeD_A1434)

GAT GGT GAT Oxaloacetate decarboxylase, subunit gamma (SD32460059)

CGC TGC TGC AIDA autotransporter (SD3246_RS06690)

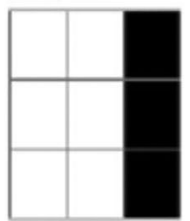

SeD_A2798 $\left({ }^{*}\right), p t s A(+)$ (Phosphoenolpyruvate) protein phosphoransferase)

SeDA41036 $\left(^{*}\right)$, WaaY ( $\left.¥\right)$

(LPS core biosynthesis protein)

SeD_A3813(*), tufB (§)

(Elongation Factor Tu)

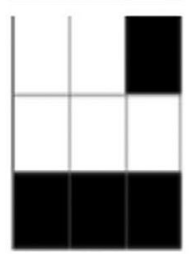

Motility

Presence of Vi antigen

Biofilm production

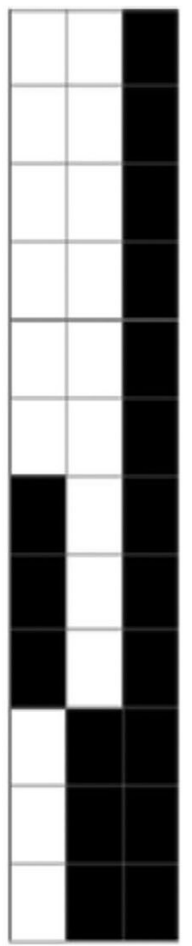

Glycerol

Fructose

Mannose

Sorbitol

$\mathrm{N}$-acetylglucosamine

Melibiose

D-xylose

L-rhamnose

Potassium 5-cetogluconate

Dulcitol

D-maltose

D-mannitol
Figure. Genomic and phenotypic characteristics of Salmonella enterica serotype Dublin isolates Str.2011, Str.2014, and Str.2015 from a 74-year-old woman who had recurrent infections involving hip joint prostheses, France. A) Tree constructed after mapping the sequences of the isolates to reference genomes of Salmonella Dublin strains CT_020221853 and SNP 3246 (GenBank accession nos. CP001144.1 and CM001151.1, respectively). The 4 nonsynonymous SNPs and the corresponding coding genes are shown. Scale bar indicates genetic distance. B) Genes in Str.2011 and altered in Str.2014 and Str.2015 (reference strain Salmonella Dublin CT_02021853) are indicated by black and white boxes, respectively. Positive phenotypic tests are indicated as black boxes. For carbohydrate metabolism, only carbohydrates used by Str.2011 and not used by Str.2014 and/or Str.2015 are shown. *Gene ID annotation based on reference strain Salmonella Dublin str. CT_02021853 (GenBank accession no. CP001144.1). Genetic events found: †14-bp deletion; ‡16-bp insertion; §790-bp deletion (total gene deletion). SNP, singlenucleotide polymorphism. 
as similarly reported for Salmonella Enteritidis isolates recovered from the bloodstream of a severely immunocompromised patient, years after the initial infection (4). Changes in carbon source availability are known to affect virulence gene regulators. In the presence/absence of a specific carbon source, specific virulence genes can be turned on or switched off, enabling pathogens to adapt to their new niche (9). Because LPS and flagellin are potent triggers of the inflammatory response, LPS alterations and the loss of flagellin expression (15) might have limited the host's innate immune response to Salmonella Dublin and facilitated its persistence at the implant interface.

\section{Conclusions}

This clinical case and other recent reports of patients with chronic infections highlight the remarkable adaptability of pathogens to a new niche. More specifically, these results show that the process of patho-adaptation of Salmonella serotypes may be extremely rapid and relies on mechanisms of genomic reshaping reminiscent of those found during the evolution of this pathogen in contact with humans. Enhanced awareness is warranted for Salmonella Dublin, especially in the elderly bearing prostheses.

\section{Acknowledgments}

We thank Vincent Enouf and Laetitia Fabre for processing the whole-genome sequencing and microbiological information, respectively. We are grateful to all the microbiological laboratories that participated in the human Salmonella network for processing the isolates.

This work was supported by the French National Reference Center for Escherichia coli, Shigella, and Salmonella, co-funded by Santé Publique France and belonging to the Integrative Biology of Emerging Infectious Diseases Laboratory of Excellence funded by the French Government Investissement d'Avenir program (grant no. ANR-10-LABX-62-IBEID).

Genomics sequence assemblies used in this project are available online on the National Center for Biotechnology Information network under accession no. PRJNA433355 (available at http://www.ncbi.nlm.nih.gov/bioproject/433355).

\section{About the Author}

Ms. El Sayed is a postgraduate fellow in clinical bacteriology of Ambroise Paré Teaching Hospital in Paris. Her research interests include bacteria adaptation and epidemiology in bone and joint infections context.

\section{References}

1. Harvey RR, Friedman CR, Crim SM, Judd M, Barrett KA, Tolar B, et al. Epidemiology of Salmonella enterica serotype Dublin infections among humans, United States, 1968-2013. Emerg Infect Dis. 2017;23:1493-501. http://dx.doi.org/10.3201/eid2309.170136
2. Crump JA, Sjölund-Karlsson M, Gordon MA, Parry CM. Epidemiology, clinical presentation, laboratory diagnosis, antimicrobial resistance, and antimicrobial management of invasive Salmonella infections. Clin Microbiol Rev. 2015;28:90137. http://dx.doi.org/10.1128/CMR.00002-15

3. McClelland M, Sanderson KE, Clifton SW, Latreille P, Porwollik S, Sabo A, et al. Comparison of genome degradation in Paratyphi A and Typhi, human-restricted serovars of Salmonella enterica that cause typhoid. Nat Genet. 2004;36:1268-74. http://dx.doi.org/10.1038/ng1470

4. Klemm EJ, Gkrania-Klotsas E, Hadfield J, Forbester JL, Harris SR, Hale C, et al. Emergence of host-adapted Salmonella Enteritidis through rapid evolution in an immunocompromised host. Nat Microbiol. 2016;1:1-6. http://dx.doi.org/10.1038/nmicrobiol.2015.23

5. Stepanović S, Cirković I, Ranin L, Svabić-Vlahović M. Biofilm formation by Salmonella spp. and Listeria monocytogenes on plastic surface. Lett Appl Microbiol. 2004;38:428-32. http://dx.doi. org/10.1111/j.1472-765X.2004.01513.x

6. Kuijpers LMF, Le Hello S, Fawal N, Fabre L, Tourdjman M, Dufour M, et al. Genomic analysis of Salmonella enterica serotype Paratyphi A during an outbreak in Cambodia, 2013-2015. Microb Genom. 2016;2:e00092. http://dx.doi.org/10.1099/mgen.0.000092

7. Besemer J, Lomsadze A, Borodovsky M. GeneMarkS: a selftraining method for prediction of gene starts in microbial genomes. Implications for finding sequence motifs in regulatory regions. Nucleic Acids Res. 2001;29:2607-18. http://dx.doi.org/10.1093/ nar/29.12.2607

8. Camacho C, Coulouris G, Avagyan V, Ma N, Papadopoulos J, Bealer K, et al. BLAST+: architecture and applications. BMC Bioinformatics. 2009;10:421. http://dx.doi.org/10.1186/1471-2105-10-421

9. Mitchell WJ. The phosphoenolpyruvate-dependent phosphotransferase system: a central feature of carbohydrate accumulation by enteric bacteria. Microbiol Sci. 1985;2:330-4, 339 .

10. Deditius JA, Felgner S, Spöring I, Kühne C, Frahm M, Rohde M, et al. Characterization of novel factors involved in swimming and swarming motility in Salmonella enterica serovar Typhimurium. PLoS One. 2015;10:e0135351. http://dx.doi.org/10.1371/ journal.pone. 0135351

11. Raetz CRH, Whitfield C. Lipopolysaccharide endotoxins. Annu Rev Biochem. 2002;71:635-700. http://dx.doi.org/10.1146/ annurev.biochem.71.110601.135414

12. Hughes D. Both genes for EF-Tu in Salmonella Typhimurium are individually dispensable for growth. J Mol Biol. 1990;215:41-51. http://dx.doi.org/10.1016/S0022-2836(05)80093-2

13. Crawford RW, Rosales-Reyes R, Ramírez-Aguilar ML, Chapa-Azuela O, Alpuche-Aranda C, Gunn JS. Gallstones play a significant role in Salmonella spp. gallbladder colonization and carriage. Proc Natl Acad Sci U S A. 2010;107:4353-8. http://dx.doi.org/10.1073/pnas.1000862107

14. McConoughey SJ, Howlin R, Granger JF, Manring MM, Calhoun JH, Shirtliff M, et al. Biofilms in periprosthetic orthopedic infections. Future Microbiol. 2014;9:987-1007. http://dx.doi.org/10.2217/fmb.14.64

15. Yim L, Sasías S, Martínez A, Betancor L, Estevez V, Scavone P, et al. Repression of flagella is a common trait in field isolates of Salmonella enterica serovar Dublin and is associated with invasive human infections. Infect Immun. 2014;82:1465-76. http://dx.doi.org/10.1128/IAI.01336-13

Address for correspondence: Simon Le Hello, Institut Pasteur, Unité de Recherche et d'Expertise des Bactéries Pathogènes Entériques, 28, rue du Docteur Roux, 75724 Paris CEDEX 15, France; email: simon. le-hello@pasteur.fr; Faten El Sayed, UFR des Sciences de la Santé Simone Veil, UVSQ, 2, avenue Source de la Bièvre, 78180 Montigny-leBretonneux, France; email: faten.elsayed@aphp.fr 